\title{
Estudio de disponibilidad léxica en el ámbito de las matemáticas
}

\author{
Lexical availability study in the field of mathematics
}

\author{
Anita Ferreira C. ${ }^{1}$, Pedro Salcedo L. $^{2}$ y María del Valle L. ${ }^{3}$ \\ ${ }^{1}$ Facultad de Humanidades y Arte, Departamento de Español, Universidad de Concepción, Chile. Correo \\ electrónico: aferreir@udec.cl \\ ${ }^{2}$ Facultad de Educación, Departamento de Metodología de la Investigación e Informática Educacional, \\ Universidad de Concepción, Chile. Correo electrónico: psalcedo@udec.cl \\ ${ }^{3}$ Facultad de Educación, Departamento de Curriculum e Instrucción, Universidad de Concepción, Chile. \\ Correo electrónico: mdelvall@udec.cl
}

La disponibilidad léxica (DL) es el área de investigación lingüística que tiene como objetivo la recogida y el posterior análisis del léxico disponible en una determinada comunidad de hablantes. En este estudio, se presenta los resultados de un proyecto de investigación, que tiene como objetivos cuantificar y describir el léxico disponible en matemáticas de los alumnos de enseñanza media de la ciudad de Concepción-Chile. Para este fin, se utiliza el modelo de disponibilidad léxica a través de un análisis cuantitativo, considerando tres índices: promedio de respuestas (XR), total de palabras diferentes (PD), y el índice de cohesión (IC). La muestra está constituida por 1557 alumnos de la ciudad de Concepción-Chile. Los resultados evidencian un crecimiento del léxico disponible por parte de los alumnos a medida que estos aumentan su nivel de escolaridad.

Palabras clave: léxico en Matemáticas, disponibilidad léxica en Matemáticas

Lexical availability (LA) is the area of linguistic research that aims at the collection and subsequent analysis of the available lexicon of a given speech community. In this study we present the results of a research project, which has among its general objectives to quantify and describe the lexicon in high school math students from the city of Concepción-Chile. To this end, the lexical availability model will be used, through a quantitative analysis, considering three indexes: response average (XR), total of different words (PD), and cohesion index (CI). The sample consists of 1,557 students in Concepción. The results show an increase of vocabulary available for the students as they increase their level of education. This was also observed in students of educational institutions of higher socioeconomic status.

Key words: Vocabulary in Mathematics, Lexical Availability in Mathematics

\section{INTRODUCCIÓN}

La disponibilidad léxica (DL) es el área de investigación lingüística que tiene como objetivo la recogida y el posterior análisis del léxico disponible de una determinada 
comunidad de hablantes. Se entiende por léxico disponible como aquellas palabras que un hablante puede activar inmediatamente en su memoria, según las necesidades derivadas de la producción lingüística.

Existe evidencia empírica de que los alumnos de enseñanza básica y media no tienen un léxico disponible suficiente (Echeverría et al. 1987; Echeverría 1991; Valencia y Echeverría 1999; Urzúa et al. 2006), lo que inevitablemente les trae problemas a la hora de leer y comprender un texto (Giammatteo et al. 2002; Giammateo y Albano 2006), o entender lo que un profesor intenta comunicar, especialmente cuando se trata de problemas con enunciado, bastante frecuentes en el área de las matemáticas. Las investigaciones en disponibilidad léxica posibilitan conocer el vocabulario disponible de grupos de hablantes específicos y contrastar los léxicos disponibles con otros grupos de hablantes de una misma lengua. Esto permite planificar y desarrollar un programa de mejoramiento de las competencias léxicas de los hablantes en ámbitos específicos como las matemáticas.

Sobre la base de lo planteado por los autores Giammateo y Albano (2006), se explica que las carencias del léxico y su relación con los procesos de lectura y escritura son tan profundas que los estudiantes no pueden comprender correctamente lo que están leyendo, impidiendo describir sus pensamientos y reflexiones. Cabe preguntarse entonces, ¿cuál es el léxico que tienen los alumnos?, ¿es el apropiado para comprender un texto y al profesor?, ¿qué sucederá con un tema especializado?, ¿cuánto léxico se requiere para que un estudiante pueda comunicarse con efectividad en un tema especializado?, ¿¿será posible determinar el léxico de cada alumno en un tema específico y elegir estrategias de enseñanza-aprendizaje adecuadas para aumentarlo? En consonancia con estas interrogantes, en este trabajo se presenta los resultados de un proyecto de investigación FONDECYT 1120911, el cual tiene entre sus objetivos generales, cuantificar y describir el léxico en matemáticas de los alumnos de enseñanza media de la ciudad de Concepción de Chile, en cuatro centros de interés (números, álgebra, geometría, y probabilidades, datos y azar/estadística). Para lo anterior, se utilizará el modelo de disponibilidad léxica, a través de un análisis cuantitativo, considerando tres índices: promedio de respuestas (XR), total de palabras diferentes (PD), y el índice de cohesión (IC).

El artículo está organizado en las siguientes secciones: en la sección 2, nos referiremos a los fundamentos teóricos en materia de disponibilidad léxica y del discurso especializado de las matemáticas. En la sección 3, se aborda el estudio descriptivo cuantitativo sobre la disponibilidad léxica matemática en estudiantes de enseñanza media de Concepción. En la sección 4, presentaremos los resultados generales y específicos de este estudio. Finalmente, señalaremos algunos comentarios finales y proyecciones sobre los avances y logros obtenidos en materia de disponibilidad léxica en la investigación realizada.

\section{FUNDAMENTACIÓN TEÓRICA}

\subsection{Disponibilidad Léxica}

La disponibilidad léxica como campo de estudio comienza a desarrollarse en los años 50. Según López Morales (1993), el primer lingüista en separar palabras frecuentes de palabras disponibles (o temáticas) fue el francés René Michéa (1953). Las 
palabras frecuentes se pueden encontrar en cualquier texto con un número moderado de páginas y sin importar su contenido, como adjetivos, verbos y sustantivos comunes (o nombres muy generales); en cambio las palabras disponibles se relacionan con cierto tema y son, en su mayoría, palabras concretas (López Morales 1993).

El léxico disponible se obtiene a través de la aplicación de encuestas, en las cuales por medio de un estímulo denominado "centro de interés" se intenta que el informante explicite su lexicón mental. Para este fin, los sujetos de la muestra deben crear listas de palabras en un tiempo determinado de dos minutos por cada centro de interés.

Uno de los primeros trabajos sobre disponibilidad léxica en el español como lengua materna (L1) fue llevado a cabo por el lingüista puertorriqueño Humberto López Morales (1973), referido a los escolares de San Juan- Puerto Rico. Actualmente, Humberto López Morales dirige y coordina el Proyecto Panhispánico sobre léxico disponible, Dispolex (http://www.dispolex.com/info/el-proyecto-panhispanico), cuyo objetivo central es elaborar diccionarios de disponibilidad léxica para las diversas zonas geográficas del mundo hispánico. En el contexto de dicho proyecto se ha llevado cabo una serie de investigaciones tanto en España como en Hispanoamérica. Como por ejemplo, el estudio de Saralegui y Tabernero (2008), quienes configuran un diccionario de léxico disponible de la ciudad de Navarra. Por su parte, Ahumada (2006) determina el léxico disponible de los estudiantes de la ciudad de Jaén. Con experiencias similares se determina el léxico disponible en República Dominicana (Alba, 1995), en Aragón, (Arnal, 2004), en Málaga, (Ávila, 2006). En Chile, Echeverría et al. (1987), Echeverría (1991), y Valencia y Echeverría (1999) investigan sobre el léxico disponible en estudiantes de educación básica y media.

Si bien existen avances importantes en el léxico disponible panhispánico general, son escasos los estudios en lo referido a la disponibilidad léxica en contextos específicos como las matemáticas. Uno de los estudios más interesantes es el realizado por Max Echeverría (1991). En este, se aplicó una encuesta de disponibilidad léxica a los alumnos y docentes de la carrera Ingeniería Civil Matemática perteneciente a la Facultad de Ciencias Físicas y Matemáticas de la Universidad de Concepción. La investigación tuvo como objetivo saber cuánto y cuál es el léxico disponible de alumnos y docentes de la carrera Ingeniería Civil Matemática en centros de interés directamente relacionados con las materias que en esta carrera se tratan. El criterio de selección para la delimitación de los centros de interés se basó en los temas que debe manejar un alumno que estudie para ser Ingeniero Civil Matemático: Cálculo, Álgebra, Estadística, Física, Ecuación y Geometría. Los resultados de esta investigación ponen de manifiesto que existe un crecimiento en el léxico disponible por parte de los estudiantes a lo largo de sus años de estudios. Por otro lado, los docentes siempre logran un mayor promedio de respuestas que los estudiantes en cada centro de interés.

\subsection{Lectura, comprensión y resolución de problemas en el discurso especializado de las matemáticas}

La Resolución de Problemas por sí sola constituye un campo de estudio en la Didáctica de la Matemática y cualquiera sea el aporte que se haga en este contexto, comprender el enunciado de un problema es el punto de partida para alcanzar su respuesta. Numerosos investigadores y estudiosos de la Resolución de Problemas se refieren a la comprensión lectora y se admite que para muchos matemáticos es más fácil escribir y establecer relaciones utilizando el lenguaje simbólico, propio de la Matemática que expresar estas ideas dialógicamente. Así entonces Polya (1949) ya 
postulaba que en la resolución de un problema lo primero que se debía hacer era leer comprensivamente su enunciado. Es más, él consideraba que este acto de comprensión se alcanzaba ejecutando las siguientes acciones: repetir el enunciado; separar las partes del enunciado, definir la incógnita y finalmente detenerse en considerar las condiciones en que el problema se daba, es decir, "el contexto". Años más tarde, Schoenfeld (1985) desestimó las ideas de Polya por considerar que éste nunca puso a prueba sus ideas respecto de la actividad cognitiva que se desarrolla cuando una persona resuelve un problema, por lo tanto no era posible aceptar con un cierto nivel de confianza lo que había postulado. Sin embargo, muchos otros estudiosos de la Resolución de Problemas (Bransford y Stein 1984; Labarrere 1987; Barrantes 2006), coincidieron en considerar a la lectura comprensiva un elemento imprescindible a la hora de resolver problemas agregando algunos de ellos, a lo ya postulado por Polya, el uso de esquemas para mejorar la comprensión del enunciado de un problema. El aporte de Schoenfeld (1985) fue indagar entre los profesores que se dedican a resolver problemas y llegó a la conclusión que la resolución de problemas no es sólo heurística si no que hay que tomar en cuenta otros factores sin olvidar que constituye una habilidad asociada al pensamiento matemático y al razonamiento lógico.

\section{Diseño DE LA INVESTIGACIÓN}

\subsection{Problema}

En lo referido al aprendizaje de las matemáticas se necesita usar un léxico especializado, que debe ser incorporado por los alumnos en el proceso de enseñanzaaprendizaje. Por tal motivo, cabe preguntarse si es posible cuantificar y describir el léxico de los alumnos de enseñanza media en dicha área científica, en centros de interés como los números, el álgebra, la geometría, y probabilidades, datos y azar. Para ello, se utilizará el modelo de disponibilidad léxica, a través de un análisis cuantitativo, considerando tres índices: promedio de respuestas (XR), total de palabras diferentes (PD), y el índice de cohesión (IC).

\subsection{Objetivos}

La investigación tiene como objetivos cuantificar y describir el léxico disponible en matemáticas que presenta una muestra de estudiantes de enseñanza media de Concepción, Chile.

\subsection{Hipótesis de la Investigación}

Se plantea como hipótesis de investigación que “el promedio del léxico disponible de los alumnos aumenta en la medida que suben en grado”, así también se busca probar que "el léxico es mayor en tipos de colegios de un estrato socioeconómico alto".

\subsection{Metodología de la Investigación}

\subsubsection{Muestra}

Los sujetos que se han encuestado en esta investigación corresponden a 1557 alumnos de Concepción-Chile: 893 mujeres y 664 hombres, provenientes de tres tipos 
de colegios (Municipal, Subvencionado y Particular) y cuatro niveles educativos de la enseñanza media (primeros, segundos, terceros y cuartos medios). En la Tabla 1 se detalla la muestra acorde con el nivel educativo y tipo de colegio.

Tabla 1: Tamaño de la muestra según tipo de establecimiento y nivel educativo

\begin{tabular}{|l|c|c|c|c|}
\hline $\begin{array}{c}\text { Nivel } \\
\text { (enseñanza media) }\end{array}$ & Total & Municipal & Subvencionados & Particulares \\
\hline Primeros & 453 & 148 & 208 & 97 \\
\hline Segundos & 376 & 131 & 162 & 83 \\
\hline Terceros & 391 & 143 & 171 & 77 \\
\hline Cuartos & 337 & 114 & 183 & 40 \\
\hline
\end{tabular}

\subsubsection{Variables}

Como en este estudio se busca, junto con describir el léxico, observar las diferencias de riqueza léxica en una prueba de DL por parte de los diferentes cursos y colegios encuestados, las variables corresponden al tipo de establecimiento y nivel educativo de los alumnos.

Se asume aquí el supuesto de asociación entre tipo de colegio y nivel socioeconómico en lo que respecta a estudiantes que concurren a colegios municipalizados, cuyo nivel socioeconómico es significativamente menor que el nivel presentado por los estudiantes que concurren a colegios particulares pagados.

\subsection{Instrumento de Investigación}

\subsubsection{Los centros de interés}

Los centros de interés fueron seleccionados de acuerdo a los ejes temáticos definidos por el Ministerio de Educación Chileno para la asignatura de matemáticas.

Las matemáticas es una disciplina cuya construcción empírica e inductiva surge de la necesidad y el deseo de responder y resolver situaciones provenientes de los más variados ámbitos, tanto de las matemáticas mismas como del mundo de las ciencias naturales, sociales, del arte y la tecnología. Los aprendizajes y el conocimiento matemático que conforman los objetivos fundamentales y contenidos mínimos obligatorios del sector fueron organizados, de acuerdo con una progresión ordenada, en cuatro ejes que articulan la experiencia formativa de alumnas y alumnos a lo largo de los años escolares. Se especificaron los siguientes centros de interés:

Números: Constituye el eje del currículo matemático para la enseñanza básica y media. Incluye los aprendizajes referidos a la cantidad y el número, las operaciones aritméticas, los diferentes sistemas numéricos, sus propiedades y los problemas provenientes de la vida cotidiana, de otras disciplinas y de la matemática misma. Se organiza en torno a los diferentes ámbitos y sistemas numéricos.

Álgebra: Este eje introduce el uso de símbolos para representar y operar con cantidades. Se inicia en quinto grado, mediante la expresión de relaciones generales y abstractas de la aritmética y la medición, que son parte de los aprendizajes de este nivel y anteriores. "El orden de los factores no altera el producto", "qué número sumado 
con 3 tiene como resultado 9", son situaciones que permiten poner en contacto con el lenguaje algebraico a cada estudiante desde los primeros niveles del currículo escolar. El álgebra provee de un lenguaje a la matemática, por ende, contribuye a, y se nutre del desarrollo de los ejes de números, geometría, y probabilidades, datos y azar. Este eje introduce también el concepto de función y el estudio de algunas de ellas en particular.

Geometría: Este eje se orienta, inicialmente, al desarrollo de la imaginación espacial, al conocimiento de objetos geométricos básicos y algunas de sus propiedades. En particular propone relacionar formas geométricas en dos y tres dimensiones, la construcción de figuras y de transformaciones de figuras. Se introduce la noción de medición en figuras planas. Este eje se relaciona con el de números, a partir de la medición y la representación, en el plano cartesiano, de puntos y figuras; con el de álgebra, y probabilidades, datos y azar, la relación se establece mediante el uso de fórmulas y luego la representación gráfica de funciones y de distribución de datos.

Probabilidades, datos y azar: Este eje introduce el tratamiento de datos y modelos para el razonamiento en situaciones de incerteza. El tratamiento de datos estadísticos se inicia en primero básico y el azar a partir de quinto. Incluye los conocimientos y las capacidades para recolectar, organizar, representar y analizar datos. Provee de modelos para realizar inferencias a partir de información muestral en variados contextos, además del estudio e interpretación de situaciones en las que interviene el azar. Desde la Educación Básica se propone desarrollar habilidades de lectura, análisis crítico e interpretación de información presentada en tablas y gráficos. En Educación Media, el estudio de Probabilidades, Datos y Azar se propone desarrollar conceptos y técnicas propias de la estadística y la teoría de probabilidades que permitan realizar inferencias a partir de información de naturaleza estadística y distinguir entre los fenómenos aleatorios y los deterministas.

\subsubsection{El Test de Disponibilidad Léxica}

La prueba se estructura en dos partes, la primera presenta un área de identificación, en la cual los sujetos debían anotar su nombre, edad, sexo y curso en el que estudian. En la segunda parte, los sujetos disponen de dos minutos para elaborar una lista de palabras por cada centro de interés.

\subsection{Procesamiento de los datos}

La información se analizó considerando los tres índices, que más aportan a la determinación de la riqueza léxica de los sujetos encuestados: el promedio de respuestas (XR), el total de palabras diferentes (PD) y el índice de cohesión (IC). El promedio de respuestas (XR) indica cuántas son las palabras que, en promedio, poseen los sujetos para su comunicación en el ámbito de conocimiento que explora el centro de interés respectivo. El total de palabras diferentes (PD) es un índice que da cuenta del total de palabras conocidas por el grupo muestral. El índice de cohesión (IC) es un indicador del grado de coincidencia en las respuestas, es decir, del léxico de un determinado centro de interés. Esto nos permite interpretar más adecuadamente los datos de los índices anteriores, pues el grado de homogeneidad en el uso del léxico está en proporción directa con el grado de disponibilidad que ese léxico tiene para todos los sujetos como conjunto. 
Para validar los datos se tomaron las siguientes decisiones: digitar todo en mayúscula, anotar en singular sustantivos y adjetivos, remitir los diminutivos a la forma primitiva, remitir todas las formas verbales al infinitivo, excepto gerundio y participio. Finalmente, el material se sometió a un procesamiento computacional para el cálculo de índices y frecuencias, a través del software Lexmath. Este software permite medir en forma online el lexicón mental de un individuo, determinando automáticamente los índices y representaciones gráficas del léxico en estudio (http://www.lexmath.com, Proyecto Fondecyt 1120911; Salcedo y Del Valle 2013; Salcedo et al. 2012; Salcedo et al. 2013).

\section{ANÁLISIS DE LOS RESULTADOS}

En las siguientes tablas y gráficos presentamos los resultados de todos los centros de interés en su conjunto acorde con las variables tipo de establecimiento (municipal, subvencionado y particular) y nivel educativo (primero medio=1ero, segundo medio $=2$ do, tercero medio=3ero y cuarto medio=4to).

Luego se presenta un análisis por cada centro de interés, en cuanto al promedio de palabras, al número de palabras diferentes y a los índices de cohesión, los cuales nos han permitido visualizar de mejor forma los resultados.

En la Tabla 2 se presenta los resultados generales de la muestra, tomando en consideración todos los centros de interés en su conjunto. Como se puede observar tanto el promedio de palabras como el Índice de Cohesión (IC) es notablemente mayor en los colegios particulares. Las razones que pueden explicar esta diferencia entre los colegios, guardan relación con el mayor número de alumnos que constituyen la muestra en los colegios municipales y subvencionados y el menor promedio de palabras detectadas en este tipo de establecimientos. Esto evidencia una menor competencia léxica en los temas consultados. Por el contrario, en los colegios particulares, aún cuando la muestra es menor en cuanto a su tamaño, los resultados arrojan un mayor promedio de palabras, es decir, un mayor conocimiento léxico de la lengua en esta área específica en estudio, las matemáticas.

Tabla 2: Resultados generales según tipo de establecimiento y considerando todos los centros (números, álgebra, geometría y probabilidades, datos y azar.)

\begin{tabular}{|l|l|l|l|l|}
\hline Resumen & Municipales & Subvencionados & Particulares & Total \\
\hline $\mathrm{N}^{\circ}$ Palabras & 19.914 & 42.461 & 11.581 & 73.956 \\
\hline $\mathrm{N}^{\circ}$ Alumnos & 536 & 724 & 397 & 1.557 \\
\hline $\mathrm{XR}$ & 37,15 & 50,07 & 54,62 & 46,34 \\
\hline $\mathrm{NPD}$ & 1,468 & 2,377 & 1,288 & 2.866 \\
\hline $\mathrm{IC}$ & 0,02530654 & 0,21064367 & 0,042406832 & \\
\hline
\end{tabular}

Fuente: encuesta de disponibilidad léxica aplicada en Fondecyt 1120911

- $\quad \mathrm{N}^{\circ}$ PALABRAS: total de palabras que nombraron los alumnos

- XR: promedio de palabras

- $\quad$ NPD: número de palabras diferentes

- IC: índice de cohesión

En la Tabla 3 se presenta los resultados ordenados acordes con las variables tipos de establecimiento (municipales, subvencionados y particulares) y nivel 
educativo (primero medio=1ero, segundo medio=2do, tercero medio=3ero y cuarto medio=4to). Al igual que los resultados reportados en las investigaciones de Echeverría (1991) y Urzúa et al. (2006) se observa un promedio mayor de palabras acorde con el nivel educativo y tipo de establecimiento. Es decir, en todos los centros de interés analizados se evidencia un crecimiento del léxico disponible por parte de los alumnos a medida que éstos aumentan su nivel de escolaridad. Así mismo, los estudiantes de establecimientos educacionales particulares obtienen un mayor promedio de respuestas que los subvencionados y estos a su vez que los municipales en todos los centros de interés.

Tabla 3: Resultados generales según tipo de establecimiento y niveles educativos de los cursos en los cuales se aplicó la prueba de DL

\begin{tabular}{|l|c|c|c|c|c|c|c|c|c|c|c|c|}
\hline & \multicolumn{4}{|c|}{ MUNICIPALES } & \multicolumn{3}{c|}{ SUBVENCIONADOS } & \multicolumn{4}{c|}{ PARTICULARES } \\
\hline & 1 ero & 2 do & 3ero & 4 to & 1 ero & 2 do & 3ero & 4 to & 1 ero & 2 do & 3 ero & 4 to \\
\hline$N^{\circ}$ Pabras & 4543 & 4292 & 6032 & 5047 & 10253 & 10611 & 9823 & 11774 & 3073 & 2420 & 3387 & 2701 \\
\hline$N^{\circ}$ Alumnos & 148 & 131 & 143 & 114 & 208 & 162 & 171 & 183 & 97 & 83 & 77 & 40 \\
\hline XR & 30,69 & 32,76 & 42,18 & 44,27 & 43,08 & 45,74 & 49,86 & 55,54 & 47,28 & 49,39 & 58,4 & 67,53 \\
\hline NPD & 652 & 693 & 812 & 785 & 1060 & 1142 & 1159 & 1495 & 568 & 564 & 687 & 691 \\
\hline IC & 0,0471 & 0,0473 & 0,0519 & 0,0564 & 0,0406 & 0,0401 & 0,0430 & 0,0372 & 0,0832 & 0,0876 & 0,0850 & 0,0977 \\
\hline
\end{tabular}

En el Gráfico 1 se puede verificar más claramente el promedio de palabras por tipo de establecimiento. Se percibe la diferencia importante entre los colegios con menor promedio de palabras (municipales) y los de mayor número (particulares). Si se considera que, en general, se admite que el nivel socioeconómico de los estudiantes que concurren a colegios municipalizados es significativamente menor que el nivel socioeconómico de los estudiantes que concurren a colegios particulares pagados es posible afirmar que existe una fuerte incidencia entre ambas variables. Esto implicaría que los colegios de mayor nivel socioeconómico presentarían entre sus estudiantes un mayor incremento léxico en el ámbito de las matemáticas.

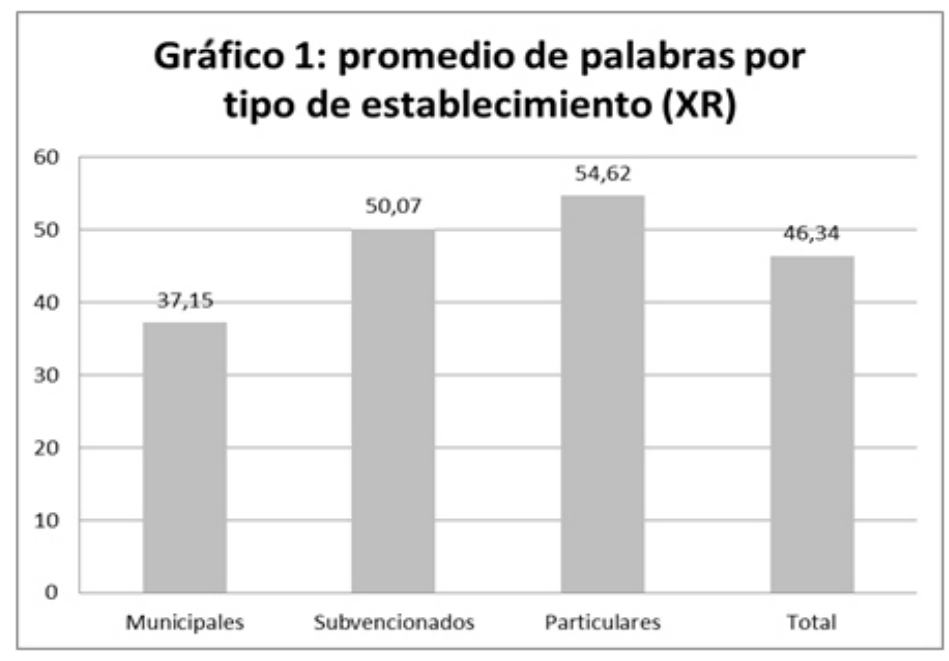


En el Gráfico 2 se ilustra de manera clara que el Indice de Cohesión de los establecimientos particulares es considerablemente mayor con respecto a los otros dos establecimientos, mostrando una similaridad en el léxico que manejan los alumnos de este tipo de establecimiento. Sin embargo, al observar el Índice de Cohesión de los establecimientos subvencionados, encontramos el caso contrario, el índice de estos es el menor. Esto puede explicarse debido a la diversidad de alumnos que han emigrado, en los últimos años, a estos colegios desde los municipales, motivados por la desconformidad de los padres en cuanto a la calidad de enseñanza entregada en dichos establecimientos.

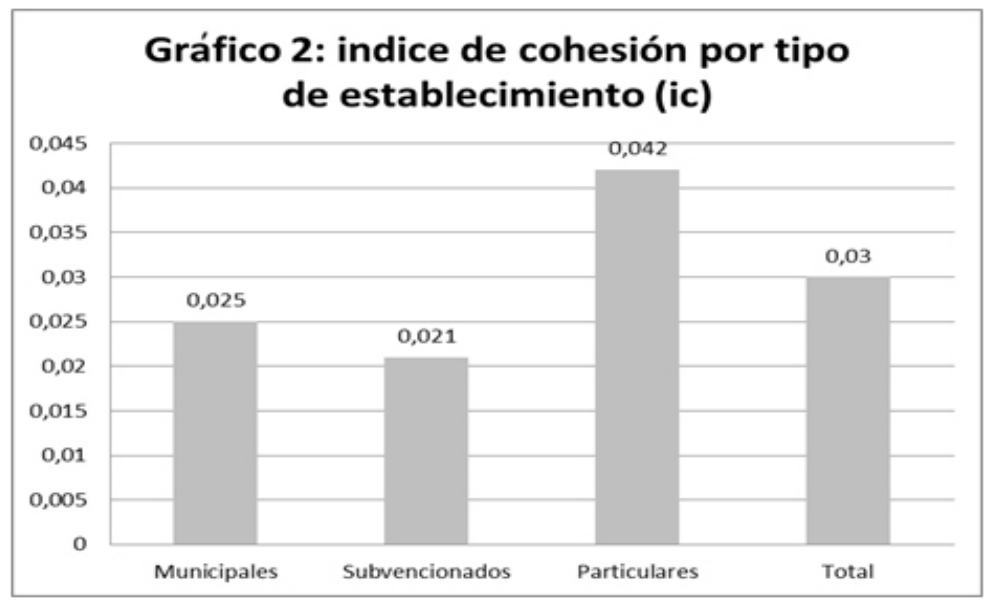

En los gráficos 3 y 4 se percibe una leve diferencia en el Índice de Cohesión entre los cuatro niveles educativos (1ero, 2do, 3ero y 4to). No así en el promedio de palabras, el cual aumenta según el nivel educativo. Se puede concluir, entonces, que los cursos adquieren un léxico similar a medida que aumenta el nivel educativo siendo irrelevante, en este caso, la variable tipo de establecimiento.

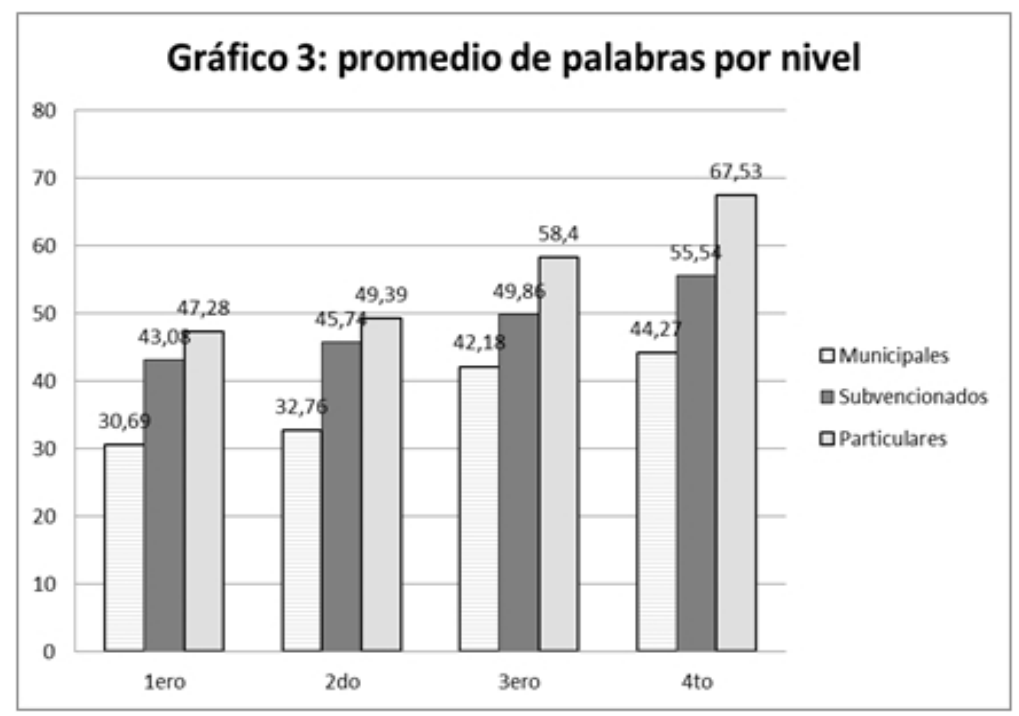




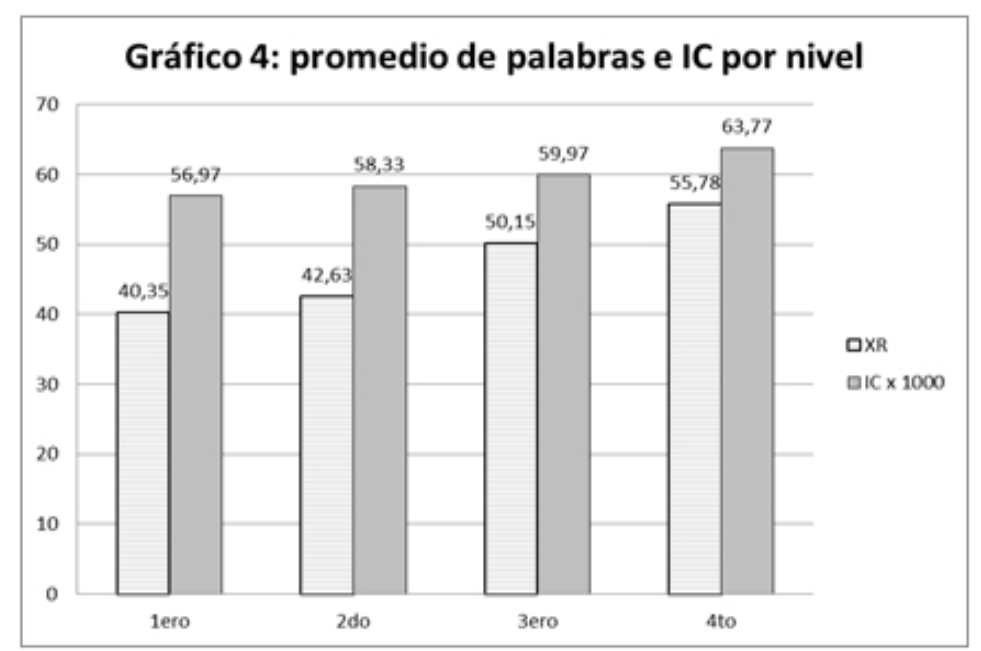

\subsection{Léxico Disponible por cada Centro de Interés}

El ordenamiento que se presenta en la Tabla 4, en que se señalan las primeras 20 palabras en orden decreciente según el IDL, muestra el léxico disponible del grupo de estudiantes en el centro de interés Álgebra acorde con el nivel educativo.

Pese a la incorporación de la temática de Álgebra en la enseñanza media, los alumnos continúan, como muestra la Tabla 4, reconociendo al vocablo "número" como uno de los más relevantes en el desarrollo de la asignatura, lo que podría significar que no han comprendido aún el nivel de abstracción entre los conceptos de número y letra. Además, en todo el trabajo algebraico propuesto en los planes y programas del Ministerio de Educación Chilena es esencial comprender la operatoria numérica para su mejor desarrollo. Esto para valorizar, resolver, identificar y determinar, entre otras, expresiones algebraicas. Todo lo anterior queda respaldado, al observar que entre los vocablos más disponibles se encuentra "letra" y "ecuación", que forman parte de la base conceptual del eje de aprendizaje Álgebra.

- Binomio, trinomio, polinomio y monomio son palabras que tienen relación directa entre sí, puesto que son las clasificaciones de expresiones algebraicas de acuerdo al número de términos de ella.

- Cuadrado de binomio y Suma por su diferencia son expresiones que aparecen en la tabla, y están relacionadas directamente con el contenido de producto notable, vocablo que también aparece dentro de las 50 palabras más disponibles de los alumnos.

- Baldor es un léxico disponible en los alumnos, encontrándose en la posición 18, lo que puede ser consecuencia del uso del libro “Álgebra” de Aurelio Baldor, en la asignatura de matemática.

- Los vocablos difícil y aburrido relacionados con la emotividad están mencionados como consecuencia de la experiencia que tienen los alumnos en el aula respecto de este centro. 
Tabla 4: Léxico Disponible en el Centro de Interés de Álgebra, considerando las primeras 20 palabras en forma descendente según ÍDL por nivel educativo

\begin{tabular}{|l|l|c|l|l|l|l|l|l|}
\hline \multicolumn{2}{|c|}{ 1RO } & \multicolumn{2}{c|}{ 2DO } & \multicolumn{2}{c|}{ 3RO } & \multicolumn{2}{c|}{ 4TO } \\
\cline { 2 - 8 } & \multicolumn{2}{|c|}{ PALABRA } & IDL & PALABRA & IDL & PALABRA & IDL & PALABRA \\
\hline 1 & NUMERO & 0,584958 & NUMERO & 0,598821 & NUMERO & 0,507292 & NUMERO & 0,429355 \\
2 & LETRA & 0,426927 & LETRA & 0,400836 & ECUACION & 0,387274 & LETRA & 0,335372 \\
3 & SUMA & 0,265327 & ECUACION & 0,252834 & LETRA & 0,254878 & ECUACION & 0,240722 \\
4 & RESTA & 0,220869 & MATEMATICA & 0,251774 & SUMA & 0,187637 & INCOGNITA & 0,169765 \\
5 & MATEMATICA & 0,219859 & MULTIPLICACION & 0,202561 & INCOGNITA & 0,184583 & SUMA & 0,163357 \\
6 & ECUACION & 0,217238 & SUMA & 0,186781 & MATEMATICA & 0,165396 & RESTA & 0,128314 \\
7 & MULTIPLICACION & 0,216526 & DIVISION & 0,168514 & RESTA & 0,148593 & BINOMIO & 0,125286 \\
8 & DIVISION & 0,170836 & BINOMIO & 0,159557 & DIVISION & 0,147159 & MULTIPLICA. & 0,117162 \\
9 & FRACCION & 0,126743 & INCOGNITA & 0,156639 & MULTIPLICA. & 0,144734 & MATEMATICA & 0,110482 \\
10 & INCOGNITA & 0,115820 & RESTA & 0,151389 & RAIZ & 0,130843 & DIVISION & 0,106213 \\
11 & BINOMIO & 0,097500 & FACTORIZACION & 0,133551 & PROBLEMA & 0,104223 & RAIZ & 0,097146 \\
12 & X & 0,082903 & FRACCION & 0,130885 & FRACCION & 0,103425 & X & 0,094778 \\
13 & PROBLEMA & 0,075027 & PROBLEMA & 0,104673 & PARABOLA & 0,094449 & FUNCION & 0,088329 \\
14 & POLINOMIO & 0,072869 & TRINOMIO & 0,079176 & X & 0,085748 & TRINOMIO & 0,084989 \\
15 & MONOMIO & 0,071857 & X & 0,077770 & ELIPSE & 0,084758 & TEOREMA & 0,082030 \\
16 & TRINOMIO & 0,069062 & RAIZ & 0,071777 & INECUACION & 0,079599 & PROBLEMA & 0,078059 \\
17 & BALDOR & 0,065789 & POLINOMIO & 0,065010 & FACTORIZA. & 0,078776 & FACTORIZA. & 0,073583 \\
18 & EJERCICIO & 0,064664 & CALCULO & 0,060451 & BINOMIO & 0,073680 & BALDOR & 0,069645 \\
19 & SIGNO & 0,063605 & C. DE BINOMIO & 0,055070 & CIRCUNFEREN & 0,070558 & PITAGORAS & 0,067570 \\
20 & FACTORIZACION & 0,062126 & D. LA INCOGNITA & 0,054748 & HIPERBOLA & 0,067557 & POTENCIA & 0,058462 \\
\hline
\end{tabular}

En el centro de interés de Geometría que se ilustra en la Tabla 5, el vocablo triángulo es el más disponible en los alumnos, debido en parte a su frecuente utilización discursiva durante todos los niveles de la enseñanza media.

- Le siguen cuadrado y ángulo en cada nivel que como la palabra anterior se presenta en los planes de estudio desde el comienzo de la incorporación de la Matemática.

- Así también se presentan las palabras suma y resta, que corresponden a dos de las cuatro operaciones básicas.

- Círculo, circunferencia, radio y diámetro son vocablos relacionados al desarrollo del estudio de la circunferencia y sus elementos.

- Además están presenten las palabras triángulo, cuadrado, ángulo rectángulo, figura, vértice, lado, rombo, pentágono, hexágono, polígono, todas ellas relacionadas con el contenido de clasificación de polígonos y sus elementos.

- $\quad$ Entre las 20 palabras, también se presentan: regla, compás y transportador, términos que son utilizados en el estudio de la geometría.

- $\quad$ Existen vocablos en la tabla que apuntan directamente al estudio de los cuerpos geométricos: cubo, vértice, arista, paralelepípedo, pirámide, volumen y cono.

Tabla 5: Léxico Disponible en el Centro de Interés de Geometría, considerando las primeras 20 palabras en forma descendente según IDL por nivel educativo

\begin{tabular}{|l|l|l|l|l|l|l|l|l|}
\hline \multirow{2}{*}{} & \multicolumn{3}{|c|}{1 RO } & \multicolumn{2}{c|}{ 2DO } & \multicolumn{2}{c|}{ 3RO } & \multicolumn{2}{c|}{ 4TO } \\
\cline { 2 - 9 } & PALABRA & IDL & PALABRA & IDL & PALABRA & IDL & PALABRA & IDL \\
\hline 1 & TRIANGULO & 0,514549 & TRIANGULO & 0,540048 & TRIANGULO & 0,606483 & TRIANGULO & 0,563377 \\
2 & CUADRADO & 0,479701 & CUADRADO & 0,419450 & CUADRADO & 0,469035 & ANGULO & 0,415743 \\
3 & ANGULO & 0,332882 & ANGULO & 0,338192 & ANGULO & 0,349261 & CUADRADO & 0,364625 \\
4 & CIRCULO & 0,273853 & CIRCULO & 0,253831 & CIRCULO & 0,298721 & CIRCULO & 0,243471 \\
5 & RECTANGULO & 0,246428 & RECTANGULO & 0,227582 & RECTANGULO & 0,267141 & RECTANGULO & 0,234961 \\
6 & FIGURA & 0,188840 & FIGURA & 0,220697 & AREA & 0,212387 & AREA & 0,230019 \\
\hline
\end{tabular}




\begin{tabular}{|l|l|l|l|l|l|l|l|l|}
\hline 7 & AREA & 0,186914 & NUMERO & 0,216611 & PERIMETRO & 0,191476 & CIRCUNFE.. & 0,184586 \\
8 & NUMERO & 0,177290 & CIRCUNFEREN & 0,189402 & NUMERO & 0,182113 & PERIMETRO & 0,175757 \\
9 & CUBO & 0,171794 & AREA & 0,178129 & VERTICE & 0,159154 & NUMERO & 0,166996 \\
10 & REGLA & 0,161630 & PERIMETRO & 0,169862 & CIRCUNFE.. & 0,156377 & PITAGORAS & 0,156926 \\
11 & PERIMETRO & 0,160330 & VERTICE & 0,126167 & FIGURA & 0,153982 & CUBO & 0,154077 \\
12 & VERTICE & 0,129337 & REGLA & 0,120464 & LADO & 0,108685 & VOLUMEN & 0,112987 \\
13 & CIRCUNFE.. & 0,100031 & THALES & 0,110383 & CUBO & 0,106153 & VERTICE & 0,107629 \\
14 & LADO & 0,096814 & RADIO & 0,105044 & REGLA & 0,099057 & EUCLIDES & 0,104848 \\
15 & COMPAS & 0,087532 & CUBO & 0,097586 & RADIO & 0,096592 & RADIO & 0,099345 \\
16 & MATEMATICA & 0,086240 & TEOREMA & 0,096460 & PENTAGONO & 0,090081 & FIGURA & 0,095955 \\
17 & TRANSPOR.. & 0,083826 & PITAGORAS & 0,094206 & ALTURA & 0,081673 & ROMBO & 0,094750 \\
18 & PIRAMIDE & 0,081479 & LADO & 0,092954 & PARALELEPI.. & 0,080815 & ALTURA & 0,091258 \\
19 & PENTAGONO & 0,077754 & MATEMATICA & 0,086357 & ARISTA & 0,080773 & ISOSCELES & 0,088573 \\
20 & SUMA & 0,073695 & HIPOTENUSA & 0,071820 & HEXAGONO & 0,076451 & LADO & 0,086483 \\
\hline
\end{tabular}

En la Tabla 6 se puede percibir el centro de interés Números, en este la palabra con mayor índice de disponibilidad léxica es “suma”, la cual es de alto uso también en los distintos niveles educativos de la enseñanza media. Le siguen las palabras: multiplicación, resta y división; que corresponden a las otras tres operaciones básicas.

Tabla 6: Léxico Disponible en el Centro de Interés de Números considerando las primeras 20 palabras en forma descendente según IDL por nivel educativo

\begin{tabular}{|c|c|c|c|c|c|c|c|c|}
\hline & \multicolumn{2}{|c|}{$1 \mathrm{RO}$} & \multicolumn{2}{|l|}{$2 \mathrm{DO}$} & \multicolumn{2}{|c|}{$3 \mathrm{RO}$} & \multicolumn{2}{|c|}{$4 \mathrm{TO}$} \\
\hline & PALABRA & IDL & PALABRA & IDL & PALABRA & IDL & PALABRA & IDL \\
\hline 1 & SUMA & 0,489033 & SUMA & 0,466356 & SUMA & 0,413112 & SUMA & 0,323768 \\
\hline 2 & RESTA & 0,418910 & RESTA & 0,406657 & RESTA & 0,364192 & MULTIPLI. & 0,296671 \\
\hline 3 & MULTIPLICA. & 0,404517 & MATEMA. & 0,366155 & MULTIPLICA. & 0,336512 & RESTA & 0,290422 \\
\hline 4 & DIVISION & 0,383665 & MULTIPLICA. & 0,362498 & DIVISION & 0,328131 & DIVISION & 0,286005 \\
\hline 5 & MATEMATI. & 0,308586 & DIVISION & 0,355213 & MATEMATICA & 0,285210 & MATEMA. & 0,194389 \\
\hline 6 & FRACCION & 0,231473 & FRACCION & 0,264680 & FRACCION & 0,149368 & NATURAL & 0,155573 \\
\hline 7 & ECUACION & 0,116771 & ECUACION & 0,196329 & ECUACION & 0,147859 & FRACCION & 0,153801 \\
\hline 8 & DECIMAL & 0,109930 & DECIMAL & 0,129363 & ALGEBRA & 0,126756 & REAL & 0,131752 \\
\hline 9 & POTENCIA & 0,106241 & ALGEBRA & 0,110677 & RAIZ & 0,123297 & RACIONAL & 0,114104 \\
\hline 10 & NUMERO & 0,098326 & RAIZ & 0,107093 & CANTIDAD & 0,108457 & ECUACION & 0,111619 \\
\hline 11 & ALGEBRA & 0,087332 & POTENCIA & 0,098862 & POTENCIA & 0,096113 & LOGARIT. & 0,110041 \\
\hline 12 & CANTIDAD & 0,078118 & CALCULADORA & 0,096552 & DECIMAL & 0,086919 & ALGEBRA & 0,103825 \\
\hline 13 & CONTAR & 0,074218 & PROBLEMA & 0,090874 & CONJUNTO & 0,084632 & ENTERO & 0,103588 \\
\hline 14 & SUMAR & 0,058975 & NUM. RACIO & 0,067146 & NUMERO & 0,079252 & CONJUNTO & 0,102602 \\
\hline 15 & CALCULADOR & 0,056658 & FISICA & 0,063228 & PROBLEMA & 0,078501 & DECIMAL & 0,098692 \\
\hline 16 & FECHA & 0,052770 & DINERO & 0,061545 & INFINITO & 0,069225 & SUMAR & 0,095431 \\
\hline 17 & ENTERO & 0,052573 & NUMERO & 0,061380 & REAL & 0,069024 & RAIZ & 0,094416 \\
\hline 18 & GEOMETRIA & 0,048200 & RACIONAL & 0,060955 & NATURAL & 0,066542 & GEOMETRIA & 0,092914 \\
\hline 19 & RESTAR & 0,047260 & NATURAL & 0,059825 & CALCULADORA & 0,066190 & RESTAR & 0,087980 \\
\hline 20 & PROBLEMA & 0,046956 & CALCULO & 0,058835 & CALCULO & 0,063002 & INFINITO & 0,085175 \\
\hline
\end{tabular}

- En relación a las cuatro operaciones básicas, se visualizan también los verbos sumar, contar, restar, dividir. Lo que nos indica que los conceptos asociados están incluidos en el aprendizaje de los alumnos como una acción, más que un contenido propiamente tal.

- Así también se puede observar que los conjuntos numéricos tienen participación en esta tabla representados por las palabras: Número, Natural, Racional, Entero, Real, Irracional y Conjunto. Esto se debe a que son contenidos que comienzan a desarrollarse durante la enseñanza básica y que son profundizados en cursos superiores.

- Se presentan también conceptos directamente relacionados con un contenido específico, como los son: positivo y negativo, par e impar, relacionados 
con el conjunto de los números enteros; fracción, décima y cifra, relacionados con el conjunto de los números racionales.

- El concepto problema se posiciona dentro de los primeros 20 vocablos en la tabla, lo que se traduce en que la mayor parte de los alumnos incorpora dentro del desarrollo del eje de Números la resolución de problemas en contexto.

- Potencia, raíz y logaritmo se hacen presente en ese orden, debido a su secuencialidad temporal.

- Que el vocablo calculadora aparezca dentro de las 20 palabras más disponibles, enfatiza que los alumnos hacen de esta herramienta un uso masivo para el desarrollo de la asignatura.

La Tabla 7 muestra los resultados del centro de interés de Probabilidades, Datos y Azar. La palabra con mayor índice de disponibilidad léxica es "probabilidad" abordada directamente de primero a tercero medio en enseñanza media. El contenido de probabilidades se enseña utilizando situaciones en contexto directamente relacionados con juegos de habilidades y azar que son conocidos de manera popular, como lo son: naipe inglés, naipe español, dominó, cachipun, cara y sello, ruleta, etc. De estas 50 palabras se distinguen 13 de ellas que tienen relación con juegos de azar y no específicamente con el centro de interés Datos y Azar. Estas palabras son: juego, dado, suerte, casino, carta, kino, dinero, poker, lotería, ruleta, apuesta, loto, ficha, máquina, bingo y juego de azar, debido a que al escuchar el nombre del centro de interés, los alumnos concentraron su atención, primordialmente en el azar. Del mismo modo, los alumnos diferencian vocablos que tienen relación con datos personales, estos son: nombre, fecha, rut, y edad, ya que fijaron su atención solo en datos. En definitiva, al comprender este centro por su significado conceptual por separado, se puede intuir que los alumnos no conocen Datos y Azar como eje de aprendizaje en la asignatura de matemáticas.

Tabla 7: Léxico disponible en el Centro de Interés de Probabilidades, Datos y Azar considerado las primeras 20 palabras en forma descendente según IDL por nivel educativo

\begin{tabular}{|c|c|c|c|c|c|c|c|c|}
\hline & \multicolumn{2}{|l|}{$1 \mathrm{RO}$} & \multicolumn{2}{|l|}{$2 \mathrm{DO}$} & \multicolumn{2}{|l|}{$3 \mathrm{RO}$} & \multicolumn{2}{|c|}{$4 \mathrm{TO}$} \\
\hline & PALABRA & IDL & PALABRA & IDL & PALABRA & IDL & PALABRA & IDL \\
\hline 1 & NUMERO & 0,262129 & PROBABILIDAD & 0,306131 & PROBABILIDAD & 0,341693 & PROBABILIDAD & 0,419798 \\
\hline 2 & PROBABILIDAD & 0,209716 & NUMERO & 0,217591 & JUEGO & 0,237707 & DADO & 0,281855 \\
\hline 3 & JUEGO & 0,189293 & JUEGO & 0,213207 & DADO & 0,230541 & JUEGO & 0,251666 \\
\hline 4 & INFORMACION & 0,166486 & INFORMACION & 0,207511 & NUMERO & 0,226334 & SUERTE & 0,211844 \\
\hline 5 & SUERTE & 0,146731 & DADO & 0,181168 & INFORMACION & 0,222437 & CASINO & 0,190739 \\
\hline 6 & DADO & 0,139488 & ESTADISTICA & 0,162636 & SUERTE & 0,164564 & CARTA & 0,190576 \\
\hline 7 & DATO & 0,133978 & GRAFICO & 0,151216 & ESTADISTICA & 0,157431 & NUMERO & 0,188382 \\
\hline 8 & GRAFICO & 0,106199 & SUERTE & 0,147649 & CARTA & 0,138425 & ESTADISTICA & 0,186025 \\
\hline 9 & MATEMATICA & 0,094779 & CASINO & 0,130133 & CASINO & 0,127824 & INFORMACION & 0,149295 \\
\hline 10 & ESTADISTICA & 0,087712 & MATEMATICA & 0,111877 & NOMBRE & 0,090517 & NOMBRE & 0,121676 \\
\hline 11 & CARTA & 0,086382 & CARTA & 0,107490 & MATEMATICA & 0,089617 & KINO & 0,102041 \\
\hline 12 & PORCENTAJE & 0,085138 & PORCENTAJE & 0,092393 & PORCENTAJE & 0,074043 & DATO & 0,099653 \\
\hline 13 & SUMA & 0,079831 & DATO & 0,077315 & GRAFICO & 0,072636 & AZAR & 0,090346 \\
\hline 14 & CASINO & 0,077058 & NOMBRE & 0,076625 & MONEDA & 0,069807 & PORCENTAJE & 0,086365 \\
\hline 15 & TABLA & 0,076988 & SUMA & 0,060346 & DATO & 0,068300 & GRAFICO & 0,083021 \\
\hline 16 & MULTIPLICACION & 0,069303 & FRACCION & 0,057434 & TABLA & 0,049131 & ENCUESTA & 0,079496 \\
\hline 17 & DIVISION & 0,065777 & TABLA & 0,056773 & PROBLEMA & 0,043778 & FECHA & 0,076249 \\
\hline 18 & AZAR & 0,060684 & RULETA & 0,044230 & POKER & 0,042605 & MONEDA & 0,069036 \\
\hline 19 & RESTA & 0,059835 & KINO & 0,043444 & FECHA & 0,042541 & POKER & 0,064783 \\
\hline 20 & FRACCION & 0,051704 & MONEDA & 0,040233 & LOTERIA & 0,041963 & MODA & 0,063556 \\
\hline
\end{tabular}




\subsection{Estadígrafos por cada Centro de Interés}

En la Tabla 8 y en el Gráfico 5 se puede observar que el mayor promedio de palabras lo presenta el Centro de Interés de Geometría y el de menor promedio lo constituye el de Probabilidades, Datos y Azar. No obstante, en el Índice de Cohesión se observa similitud entre los centros de Probabilidades, Datos, y Azar, Números y Álgebra, siendo notablemente mayor el observado en el centro de Geometría. De esto se infiere que el Centro de Geometría junto con tener un elevado promedio de palabras, presenta una similaridad alta en el léxico observado en todos los alumnos de la muestra.

Tabla 8: Estadígrafos por cada centro

\begin{tabular}{|c|c|c|c|}
\hline CENTRO & XR & NPD & IC \\
\hline Geometría & 16,11 & 1037 & 0,015535 \\
\hline Números & 12,94 & 1376 & 0,009404 \\
\hline Álgebra & 10,75 & 1422 & 0,00756 \\
\hline Datos y Azar & 7,44 & 1118 & 0,006655 \\
\hline
\end{tabular}

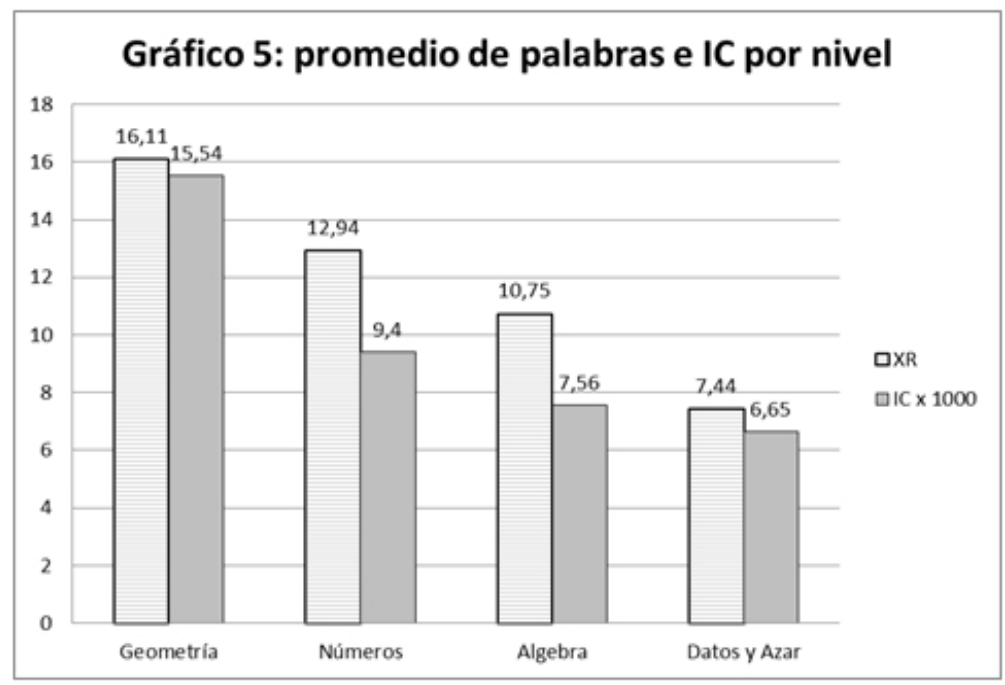

\section{CONCLUSIONES}

Según la bibliografía estudiada, el léxico disponible de los estudiantes puede considerarse como una muestra concreta y representativa de las palabras que los alumnos de cada curso y tipo de establecimiento utilizan. Los resultados evidencian de forma general un aumento en el promedio de palabras y palabras diferentes a medida que aumenta el nivel educativo y tipo de establecimiento, observándose solo una leve diferencia entre los establecimientos subvencionados y particulares. Además, los resultados arrojaron una mayor cohesión léxica en los establecimientos 
municipales que en los subvencionados, lo cual significa que el vocabulario usado en los colegios municipales es más parecido entre los alumnos que el léxico observado en los colegios subvencionados.

Entre las conclusiones más significativas se observa una fuerte similitud entre el Índices de Cohesión de los 4 niveles educativos (primero, segundo, tercero y cuarto medio). Concluyendo que los cursos adquieren el léxico de manera similar a medida que aumenta su nivel educativo. Estos resultados guardan relación con lo reportado en las investigaciones de Echeverría (1991) y Urzúa et al. (2006) .

En cuanto a los cuatro centros de interés estudiados, el de “Geometría” muestra el más alto promedio de palabras y el mayor Índice de Cohesión, lo que implica, entonces, que los alumnos de la ciudad de Concepción-Chile tienen un mayor manejo léxico en dicha área de las matemáticas y que el léxico adquirido es similar entre los alumnos encuestados.

Finalmente, los hallazgos encontrados en este estudio en cuanto al léxico disponible matemático permitirán, en el ámbito de la Lingüística Aplicada, implementar un programa de planificación lingüística conducente a lograr un mejoramiento en las competencias léxicas de los estudiantes de enseñanza media de la ciudad de Concepción- Chile, a partir de un modelo metodológico de enseñanza-aprendizaje de las matemáticas que integre el desarrollo de la lengua en contextos específicos.

\section{Agradecimientos}

Proyecto de Investigación - Fondecyt 1120911 “Disponibilidad Léxica Matemática en estudiantes de Enseñanza Media y su aplicación en Hipermedios Adaptativos”, de la Comisión Nacional de Investigación Científica y Tecnológica de Chile.

\section{OBRAS CITADAS}

Ahumada, Lara. 2006. El léxico disponible de los estudiantes de Jaén. Jaén: Universidad.

Alba, Orlando. 1995. El léxico disponible de la República Dominicana. Santo Domingo: Pontificia Universidad Católica Madre y Maestra.

Arnal, María Luisa, (coord.). 2004. Léxico disponible de Aragón. Zaragoza: Libros Pórtico.

Ávila, Antonio. 2006. Léxico disponible de los estudiantes preuniversitarios de Málaga. Málaga: Universidad.

Barrantes, Hugo. 2006. "Resolución de Problemas. El Trabajo de Allan Schoenfeld". Cuadernos de Investigación y Formación en Educación Matemática. Año 1, 1

Bransford, John y Barry Stein. 1986. Solución Ideal de Problemas. Guía para mejor pensar, aprender y crear. Barcelona: Labor.

Dispolex. 2011. "Proyecto Prehispánico de Léxico Disponible” [en línea]. Disponible en: http://www.dispolex.com

Echeverría, Max. 1991. "Crecimiento de la disponibilidad léxica en estudiantes chilenos de nivel básico y medio” Humberto López Morales (Ed.) La enseñanza del español como lengua materna. Río Piedras: Universidad de Puerto Rico. 61-78.

Echeverría, Max, María Herrera, Patricio Moreno y Francisco Pradenas. 1987.

“Disponibilidad léxica en Educación Media”. Revista de Lingüística Teórica y Aplicada 25: $55-115$.

Giammatteo, Mabel, Hilda Albano y María Basualdo. 2002. "Competencia Léxica y Aprendizaje. Interrelación de Resultados de Investigaciones realizadas en los niveles medio y superior”. Revista de Filología 33: 1-2. 
Giammatteo, Mabel e Hilda Albano. 2006. "El aprendizaje léxico en la encrucijada entre la gramática y el texto”. Revista de Filología 37.

Labarrere, Alberto. 1987. "La formación de procedimientos generales para la solución de problemas matemáticos en la escuela primaria”. Revista Ciencias Pedagógicas 14.

Lexmath. 2013. "Lexmath.com un Hipermedio Adaptativo a la disponibilidad léxica”, manual del usuario online en la dirección http://www.lexmath.com (ext. 05/01/2014).

López Morales, Humberto. 1973. Disponibilidad léxica de los escolares de San Juan. San Juan de Puerto Rico: Universidad de Puerto Rico.

López Morales, Humberto. 1978. "Frecuencia léxica, disponibilidad y programación curricular”. Boletín de la Academia Puertorriqueña de la Lengua Española 6(1): 73-86.

López Morales, Humberto. 1993. "Los estudios de disponibilidad léxica: pasado y presente”. Boletín de Filología de la Universidad de Chile XXXV:245-259.

Michéa, René. 1953. "Mots fréquents et mots disponibles. Un aspect nouveau de la statistique du langage”. Les langues modernes 47: 338-344.

Polya, George. 1945. How to solve it. Princeton: Princeton University Press.

Salcedo, Pedro y María Del Valle. 2013. "Disponibilidad Léxica Matemática en Estudiantes de Enseñanza Media de Concepción, Chile”. Atenas, Revista Científica Pedagógica 4 (21) ISSN: 1682-2749.

Salcedo, Pedro, Ferreira, Anita y Fernanda Barrientos. 2013. "A Bayesian Model for Lexical Availability of Chilean High School Students in Mathematics". Natural and Artificial Models in Computation and Biology. 245-253.

Salcedo, Pedro, Oscar Nail y Carla Arzola. 2012. "Análisis de Relaciones Semánticas del Léxico Disponible en Matemáticas en un Hipermedio Adaptativo”. XVII Congreso Internacional de Informática Educativa, TISE.

Saralegui, Carmen y Cristina Tabernero. 2008. “Aportación al proyecto panhispánico de léxico disponible: Navarra”. Actas del XXXVII Simposio Internacional de la Sociedad Española de Lingüística 745-761.

Schoenfeld, Alan. 1985. Mathematical Problem Solving. New York: Academic Press.

Urzúa Paula, Katia Sáez y Max Echeverría. 2006. “Disponibilidad Léxica Matemática. Análisis Cuantitativo y Cualitativo”. RLA 44 (2):59-76.

Valencia, Alba y Max Echeverría. 1999. Disponibilidad léxica en estudiantes chilenos. Santiago de Chile y Concepción: Universidad de Chile y Universidad de Concepción. 